\title{
NOISE CANCELLATION OVER SPATIAL REGIONS USING ADAPTIVE WAVE DOMAIN PROCESSING
}

\author{
Jihui Zhang ${ }^{1,2}$, Wen Zhang $^{1}$, Thushara D. Abhayapala ${ }^{1}$ \\ ${ }^{1}$ Research School of Engineering, The Australian National University, \\ Canberra ACT 2601, Australia \\ ${ }^{2}$ School of Electrical Engineering and Automation, Harbin Institute of Technology, \\ Harbin 150001, China
}

\begin{abstract}
This paper proposes wave-domain adaptive processing for noise cancellation within a large spatial region. We use fundamental solutions of the Helmholtz wave-equation as basis functions to express the noise field over a spatial region and show the wave-domain processing directly on the decomposition coefficients to control the entire region. A feedback control system is implemented, where only a single microphone array is placed at the boundary of the control region to measure the residual signals, and a loudspeaker array is used to generate the anti-noise signals. We develop the adaptive wave-domain filtered-x least mean square algorithm. Simulation results show that using the proposed method the noise over the entire control region can be significantly reduced with fast convergence in both free-field and reverberant environments.
\end{abstract}

Index Terms - Active noise control, Adaptive algorithm, Feedback system, Multi-channel system, Wave domain signal processing

\section{INTRODUCTION}

Active noise control (ANC), or noise cancellation, employs secondary sound sources to reduce the primary noise based on the principle of destructive interference [1]. This technique has been successfully used in several commercial applications, such as noisecancelling headphones [2], the control of noise in industrial machines [3] and active noise cancellation for automobiles [4]. Given the fact that most of the time noise is unknown and time-varying, normally adaptive filters are employed to produce anti-noise signals. Some well-known algorithms for implementation include the least-mean-square (LMS) method or its variants, such as filtered-x LMS (FxLMS), adjoint LMS and recursive LMS [5].

The technique of noise cancellation at some spatial points is simple and effective, where only one or two microphones and speakers are required to measure the residual signal and produce the anti-noise signal. A growing research interest is in creating a large-sized quiet zone for multiple listeners, which requires multiple microphones and loudspeakers to be used and is known as multichannel ANC system [6]. Both time-domain [7,8] and frequencydomain $[9,10]$ algorithms have been implemented in the multichannel ANC systems. Especially, multi-channel version of FxLMS algorithm in the frequency domain has been widely used in practice $[10,11]$. One drawback of the conventional multi-channel ANC

Thanks to Australian Research Councils Discovery Projects funding scheme (project no. DP140103412). The work of J. Zhang was sponsored by the China Scholarship Council with the Australian National University. system is that to achieve a quite zone, error microphones need to be uniformly placed inside the region to measure the residual signals so that the noise energy within the entire region can be cancelled completely.

Recently, wave-domain signal processing has been proposed to achieve sound control over large spatial regions, such as echo cancellation [12-14], room equalization for massive multichannel sound field reproduction systems [15-18] and acoustic quiet zone generation [19-21]. The principle of wave-domain signal representation is to use fundamental solutions of the Helmholtz waveequation as basis functions to express any wavefield over a spatial region. Processing directly on the decomposition coefficients therefore controls sound over a region. In [22], a wave-domain ANC system has been investigated, where one loudspeaker array and two microphone arrays, including one reference microphone array placed outside the loudspeaker array and one error microphone array placed at the boundary of the quiet zone, are adopted and the feedforward ANC system is implemented. Significant noise cancellation over the entire region of interest has been achieved.

In this paper, we propose the feedback ANC system in the wave domain using only a single error microphone array placed at the boundary of the desired quite zone to measure the residual signals and one loudspeaker array to produce anti-noise signals (see Figure 1). Adaptive wave-domain FxLMS algorithm is implemented and demonstrates good performance for noise cancellation in both free-field and reverberant environments. The rest of this paper is organized as follows. Section 2 provides the system model of the wave-domain ANC system. In Section 3, we derive adaptive wavedomain processing algorithm. In Section 4, simulations are conducted to evaluate the proposed algorithm in comparison with the conventional multi-channel ANC system. Conclusions are provided in Section 5.

\section{SYSTEM MODEL}

The noise field due to a 2D point source at an arbitrary point $\boldsymbol{x} \equiv$ $\left\{\left\|\boldsymbol{x}, \phi_{\boldsymbol{x}}\right\|\right\}$ can be represented as

$$
N(\boldsymbol{x}, k)=\sum_{m=-\infty}^{\infty} \beta_{m}(k) J_{m}(k\|\boldsymbol{x}\|) \exp \left(i m \phi_{\boldsymbol{x}}\right),
$$

where $k=2 \pi f / c$ is the wavenumber, $f$ is the frequency and $c$ the speed of sound propagation, $J_{m}(\cdot)$ is the Bessel function of order $m$ and $\exp (\cdot)$ denotes the exponential function. The wave domain coefficients $\beta_{m}(k)$ represent the noise field. 


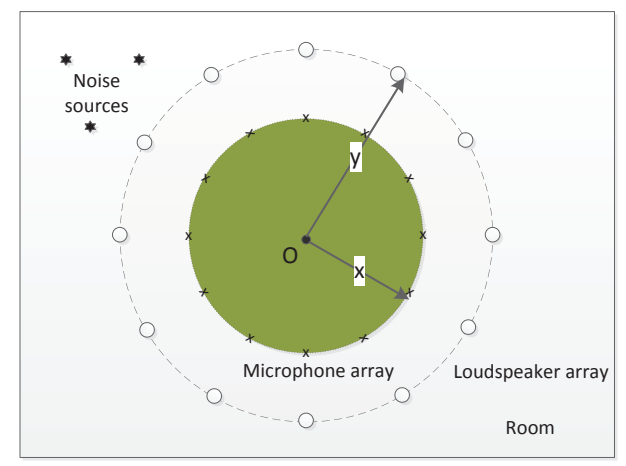

Figure 1: ANC setup with a circular quiet zone (green area) using a single microphone array to measure residual signals and a loudspeaker array to produce anti-noise signals.

The aim here is to cancel the noise completely within a circular region of interest, i.e., to create a quiet zone, using active control techniques as shown in Figure 1. Within this circular region $\|\boldsymbol{x}\| \leq$ $r$, we can use a finite number of modes to approximate the noise field [23], that is (1) reduces to

$$
N(\boldsymbol{x}, k) \approx \sum_{m=-M}^{M} \beta_{m}(k) J_{m}(k\|\boldsymbol{x}\|) \exp \left(i m \phi_{\boldsymbol{x}}\right),
$$

where $M=\lceil e k r / 2\rceil[23,24]$.

A circular array of $L$ equiangularly placed loudspeakers is used to generate the control signals. We assume a continuous loudspeaker distribution $\rho(\boldsymbol{y}, k)$, where

$$
\rho(\boldsymbol{y}, k)=\sum_{m=-\infty}^{\infty} \gamma_{m}(k) \exp \left(i m \phi_{\boldsymbol{y}}\right) .
$$

Thus, the sound field generated can be written as

$$
S(\boldsymbol{x}, k)=\int_{0}^{2 \pi} \rho(\boldsymbol{y}, k) A(\boldsymbol{x} \mid \boldsymbol{y}, k) d \phi_{\boldsymbol{y}}
$$

where $A(\boldsymbol{x} \mid \boldsymbol{y}, k)$ denotes the acoustic transfer function (ATF) between a source $\boldsymbol{y}$ and an observation point $\boldsymbol{x}$. When the observation points are inside the quit zone, the ATF can also be parameterised in the wave domain [16] as

$$
A(\boldsymbol{x} \mid \boldsymbol{y}, k)=\sum_{m=-M}^{M} a_{m}(k) J_{m}(k\|\boldsymbol{x}\|) \exp \left(i m \phi_{\boldsymbol{x}}\right),
$$

where $a_{m}(k)$ are ATF coefficients and assumed to be a prior knowledge obtained from theoretical solutions or pre-calibration.

Substituting (3) and (5) into (4) leads to the wave domain representation of the sound field generated by the control sources, that is

$$
S(\boldsymbol{x}, k) \approx \sum_{m=-M}^{M} \gamma_{m}(k) \alpha_{m}(k) J_{m}(k\|\boldsymbol{x}\|) \exp \left(i m \phi_{\boldsymbol{x}}\right),
$$

where

$$
\alpha_{m}(k)=\int_{0}^{2 \pi} a_{m}(k) \exp \left(i m \phi_{\boldsymbol{y}}\right) d \phi_{\boldsymbol{y}}
$$

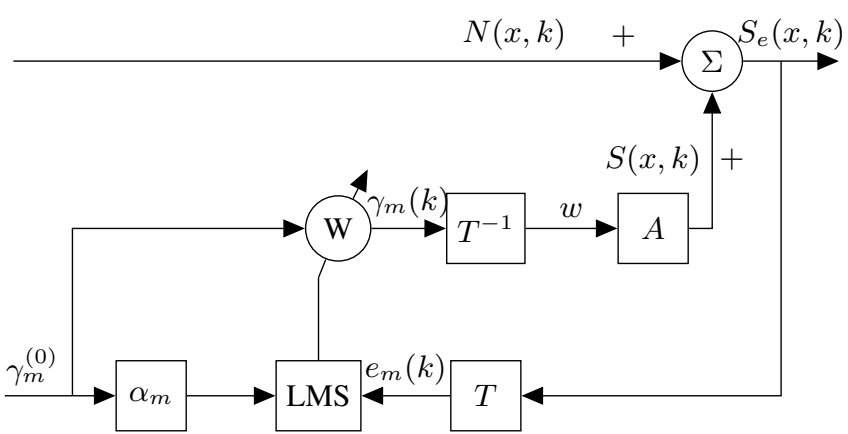

Figure 2: Block diagram of the wave-domain FxLMS algorithm for ANC. Blocks of $T$ and $T^{-1}$ represent wave-domain transformation and inverse wave-domain transformation, respectively.

For example, for sound propagation in the free field, $A(\boldsymbol{x} \mid \boldsymbol{y}, k)=$ $\frac{i}{4} H_{0}^{(1)}(k\|\boldsymbol{y}-\boldsymbol{x}\|)$ and $\alpha_{m}(k)=\frac{i}{4} H_{m}^{(1)}(k R)$, where $H_{m}^{(1)}$ denotes the first kind Hankel function of order $m$.

Wave domain noise cancellation is to design the source coefficients $\gamma_{m}(k)$ based on the ATF information $\alpha_{m}(k)$ so that the noise field $N(\boldsymbol{x}, k)$ characterised by its coefficients $\beta_{m}(k)$ is completely cancelled within the desired quiet zone. An optimal solution would be $\gamma_{m}(k)=-\beta_{m}(k) / \alpha_{m}(k)$ if a stable noise field is assumed. However, in practice the noise field is always unknown and could be time-varying, an adaptive algorithm is proposed in the next section.

\section{ADAPTIVE WAVE DOMAIN PROCESSING}

In the proposed system (Figure 1), a single circular microphone array is placed on the boundary of the quiet zone to measure the residual signals. There are $Q$ microphones equiangularly placed in the error microphone array to measure the sound field $S_{e}(\boldsymbol{x}, k)$, the superposition of the noise field and sound field generated by the control sources. The error signals in the wave domain are written as

$$
\begin{array}{r}
e_{m}(k)=\frac{1}{Q J_{m}(k r)} \sum_{q=1}^{Q} S_{e}\left(\boldsymbol{x}_{q}, k\right) \exp (-i 2 \pi m(q-1) / Q), \\
m=-M, \ldots, M .
\end{array}
$$

It is clear that the number of microphones required is $Q \geq 2 M+1$ so that all the wave domain error signals can be obtained without causing a spatial aliasing problem.

The wave domain Filtered-X Least Mean Square (WD-FxLMS) algorithm is developed in the following to update the source coefficients for cancelling the noise field over a spatial region. The residual signal in each iteration is expressed as

$$
\hat{e}_{m}(n)=\beta_{m}+\alpha_{m} \times \gamma_{m}(n),
$$

where $\gamma_{m}(n)=W_{m}(n) \gamma_{m}^{(0)}, n$ denotes the iteration step, $W_{m}(n)$ are the updated weights, and $\gamma_{m}^{(0)}$ represents an arbitrary initial estimate of the source distribution coefficients. The wavenumber $k$ has been omitted for notational simplicity.

The adaptive algorithm minimises the instantaneous squared error, $\xi_{m}^{(n)}=\left|\hat{e}_{m}(n)\right|^{2}=\hat{e}_{m}(n) \hat{e}_{m}^{*}(n)$; and adopting the steepest 


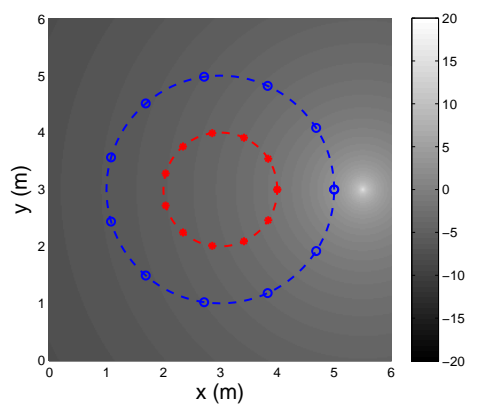

(a)

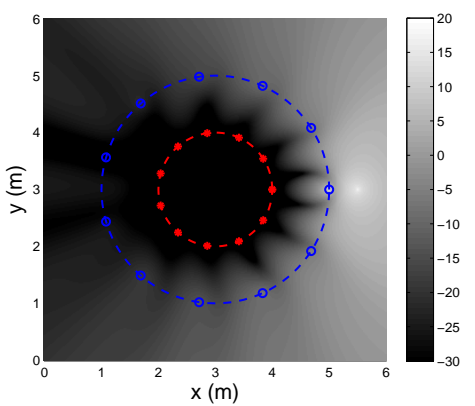

(b)

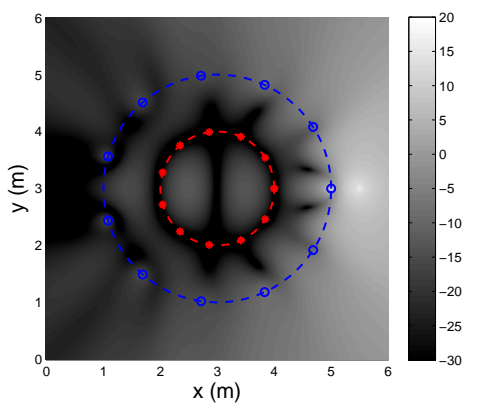

(c)

Figure 3: The results of ANC in free-field. The inner array is the microphone array, outer array is the loudspeaker array. (a) The energy of the initial noise field. (b) The residual energy after 30 iterations of WD-FxLMS. (c) The residual energy after 30 iterations of MC-FxLMS.

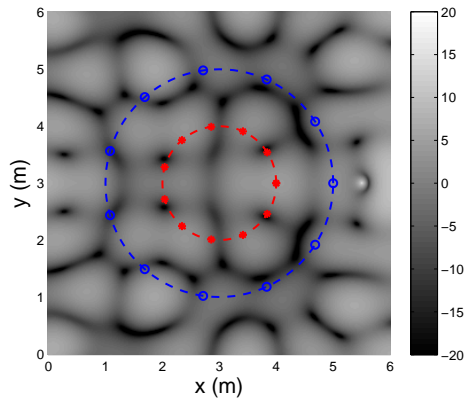

(a)

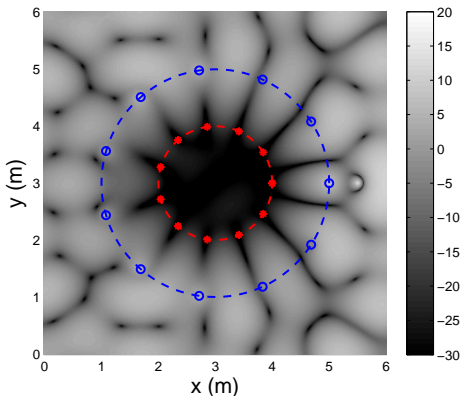

(b)

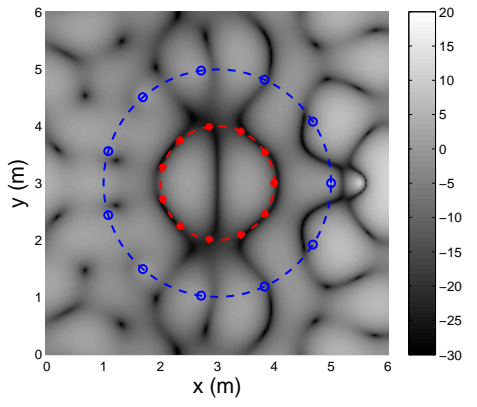

(c)

Figure 4: The results of ANC in reverberant environment. The inner array is microphone array, outer array is loudspeaker array. (a) The energy of the initial noise field. (b) The residual energy after 30 iterations of WD-FxLMS. (c) The residual energy after 30 iterations of MC-FxLMS.

descent algorithm, we have

$$
W_{m}(n+1)=W_{m}(n)-\frac{\mu}{2} \nabla \xi_{m}(n),
$$

where $\nabla \xi_{m}(n)=2 \hat{e}_{m}(n)\left[\alpha_{m} \gamma_{m}^{(0)}\right]^{*}$. Note that here we deal with complex numbers and thus use the complex LMS algorithm to update the weights [25].

Thus, we have the wave domain FxLMS algorithm,

$$
W_{m}(n+1)=W_{m}(n)-\mu \hat{e}_{m}(n)\left[\alpha_{m} \gamma_{m}^{(0)}\right]^{*}
$$

where the maximum step size $\mu_{\max }=1 /\left\|\alpha_{m} \gamma_{m}^{(0)}\right\|^{2}$ [5]. The block diagram of the algorithm is shown in Figure 2.

Given the estimated source distribution coefficients, a discrete loudspeaker array is designed to approximate the continuous source distribution. For example, we assume $L \geq 2 M+1$ loudspeakers equiangularly placed in the circular array; the $\ell$ th loudspeaker driving signal is

$$
w_{\ell}=\rho\left(\boldsymbol{y}_{\ell}, k\right) \Delta \phi
$$

where $\Delta_{\phi}=2 \pi / L$.

\section{SIMULATION}

In this section, the proposed wave domain adaptive algorithm, WDFxLMS, is evaluated through simulations of cancelling the noise field due to a 2D point source in a free-field environment and a reverberant environment, respectively. The algorithm is compared with the conventional frequency domain multichannel noise cancelling algorithm, MC-FxLMS [11]. In the simulation, the quiet zone is a circular region of radius $1 \mathrm{~m}$. We place the loudspeaker array on a circle of $R=2 \mathrm{~m}$ and the microphones are equally spaced on the boundary of the quiet zone. A signal-to-noise (SNR) ratio of $40 \mathrm{~dB}$ is added to the microphone recordings.

Case 1-Free-field Environment: Plots in Figure 3 show the results of cancelling the noise field in free-field environment using the proposed WD-FxLMS and conventional MC-FxLMS. The noise source is a $2 \mathrm{D}$ omni-directional point source located at $(5.5$, 3 ) and operates at the frequency of $200 \mathrm{~Hz}$. According to (2) and the rule of thumb [23], such a noise field within the desired quiet zone (radius of $1 \mathrm{~m}$ ) needs $m \in[-5,5]$ orthogonal modes to represent; thus, we place 11 loudspeakers and 11 microphones in each corresponding array. After 30 iterations of wave-domain adaptive processing, Figure 3(b) shows that the noise energy inside the entire quiet zone has been reduced to a very low level. Using the same loudspeaker array and microphone array setup, the conventional MC-FxLMS algorithm [11] however can only achieve noise cancellation at the microphone positions or the points very close to the microphones as shown in Figure 3(c). Inside the quiet zone, a fairly high level of noise field still exists. Figure 5 compares the convergence performance of the WD-FxLMS and MC-FxLMS algorithm, where 11 microphone recordings and measurements from 1296 points uniformly placed inside the regions are used. The WDFxLMS demonstrates fast convergence at both recording positions and at points inside the region.

Case 2-Reverberant Environment: The simulations are made 


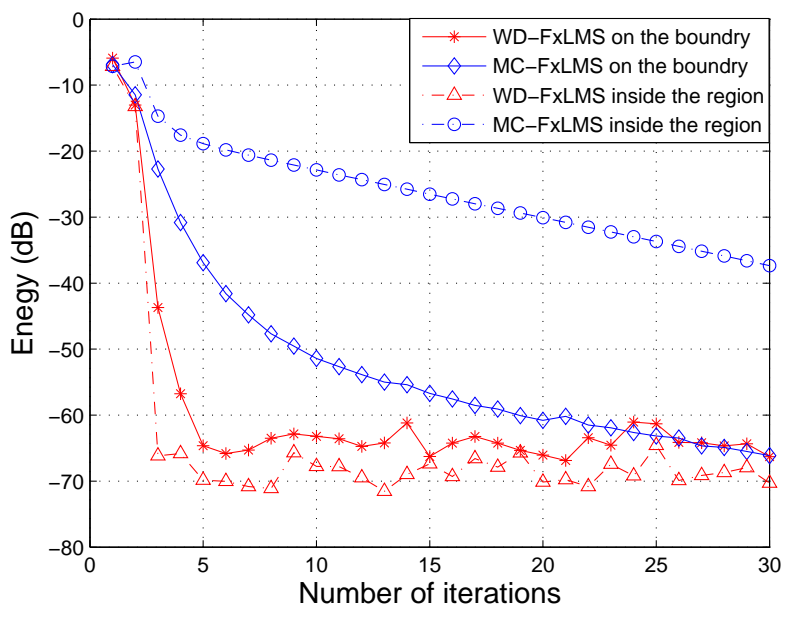

Figure 5: Comparison of convergence performance for noise cancellation using WD-FxLMS and MC-FxLMS algorithm in freefield.

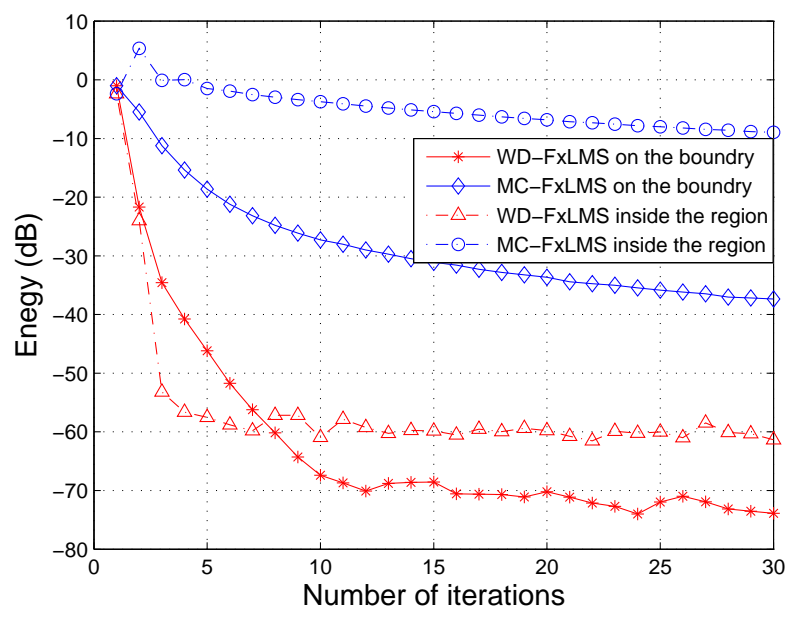

Figure 6: Comparison of convergence performance for noise cancellation using WD-FxLMS and MC-FxLMS algorithm in reverberant environment.

in a room of size $6 \mathrm{~m} \times 6 \mathrm{~m}$ and all the side walls have a reflection coefficient of 0.9 . The reflections are simulated by image source method. The origin of the loudspeaker and microphone arrays is the centre of the room. The same noise source as in the case of freefield environment is simulated. The results in Figure 4 demonstrate that the proposed WD-FxLMS algorithm can cancel the noise field completely within the desired quiet zone; while the conventional MC-FxLMS can only cancel noises at the microphone positions or the boundary of the quiet zone. Figure 6 compares the convergence performance of the WD-FxLMS and MC-FxLMS algorithm. Both can achieve noise cancellation at the recording positions, i.e., the boundary of the designed quiet zone. It can be seen clearly that the convergence rate of the proposed WD-FxLMS is much faster than that of the conventional MC-FxLMS algorithm. For all the points inside the designed region, only the wave domain adaptive processing can achieve noise energy reduction to a very low level. We can conclude that the proposed WD-FxLMS algorithm is much more effective, especially for achieving noise cancellation over a spatial region.

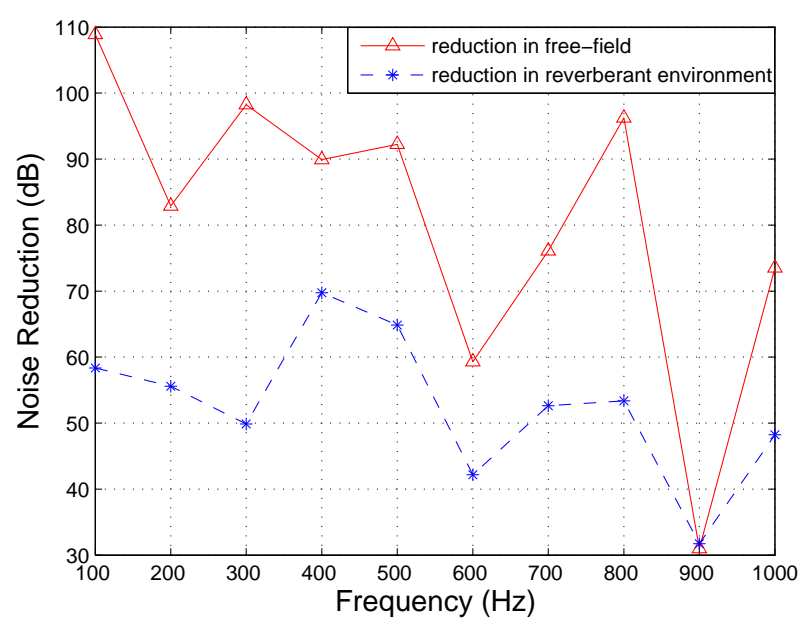

Figure 7: Broadband performance of noise reduction using WDFxLMS algorithm after 30 iterations.

We next investigate the broadband performance of the proposed algorithm. The system is designed at the frequency of $1 \mathrm{kHz}$. The noise field within a radius of $1 \mathrm{~m}$ is due to 10 noise sources uniformly distributed on a circle $2.5 \mathrm{~m}$ away from the origin and at angles $[0: 36: 324]^{\circ}$. Figure 7 shows that for all the frequencies below $1 \mathrm{kHz}$, using the WD-FxLMS algorithm, the noise can be reduced significantly within the desired quiet zone. The average noise reduction is around $80 \mathrm{~dB}$ and $50 \mathrm{~dB}$, in free-field and reverberant environment, respectively. Notice that the performance is significantly degraded at $600 \mathrm{~Hz}$ and $900 \mathrm{~Hz}$. This is due to the fact that at these particular frequencies, the Bessel functions are close to zero (i.e., $J_{m}(k r) \approx 0$ ) and the coefficient error is amplified. This problem could be avoided by placing two closely spaced microphone arrays [16].

\section{CONCLUSION}

Adaptive wave-domain processing was presented in this paper to achieve noise cancellation over a spatial region. We proposed a feedback control system using one microphone array and one loudspeaker array to measure the residual signals and produce anti-noise signals. The system were implemented in both free-field and reverberant environments and compared with the conventional multichannel ANC system. Simulation results show that the proposed adaptive wave-domain algorithm achieves significant noise reduction over the entire design region with fast convergence speed.

\section{REFERENCES}

[1] M. Bergamasco, F. D. Rossa, and L. Piroddi, "Active noise control with on-line estimation of non-Gaussian noise characteristics," Journal of Sound and Vibration, vol. 331, no. 1, pp. 27 - 40, January 2012. 
[2] S. Kuo, S. Mitra, and W.-S. Gan, "Active noise control system for headphone applications," IEEE Transactions on Control Systems Technology, vol. 14, no. 2, pp. 331-335, March 2006.

[3] R. Reddy, I. Panahi, and R. Briggs, "Hybrid FxRLS-FxNLMS adaptive algorithm for active noise control in fMRI application," IEEE Transactions on Control Systems Technology, vol. 19, no. 2, pp. 474-480, March 2011.

[4] H. Sano, T. Inoue, A. Takahashi, K. Terai, and Y. Nakamura, "Active control system for low-frequency road noise combined with an audio system," IEEE Transactions on Speech and Audio Processing, vol. 9, no. 7, pp. 755-763, October 2001.

[5] S. M. Kuo and D. R. Morgan, "Active noise control: A tutorial review," Proceedings of the IEEE, vol. 87, no. 6, pp. 943-973, June 1999.

[6] Y. Kajikawa, W.-S. Gan, and S. M. Kuo, "Recent advances on active noise control: Open issues and innovative applications," APSIPA Transactions on Signal and Information Processing, vol. 1, p. 21, April 2012.

[7] A. Barkefors, S. Berthilsson, and M. Sternad, "Extending the area silenced by active noise control using multiple loudspeakers," in Proc. IEEE ICASSP 2012, Kyoto, Japan, March 2012, pp. 325-328.

[8] M. Bouchard and S. Quednau, "Multichannel recursive-leastsquare algorithms and fast-transversal-filter algorithms for active noise control and sound reproduction systems," IEEE Transactions on Speech and Audio Processing, vol. 8, no. 5, pp. 606-618, September 2000.

[9] J. Benesty and D. Morgan, "Frequency-domain adaptive filtering revisited, generalization to the multi-channel case, and application to acoustic echo cancellation," in Proc. IEEE ICASSP 2000, vol. 2, Istanbul, Turkey, June 2000, pp. II789II792.

[10] J. Lorente, M. Ferrer, M. De Diego, and A. González, "GPU implementation of multichannel adaptive algorithms for local active noise control," IEEE/ACM Transactions on Audio, Speech, and Language Processing, vol. 22, no. 11, pp. 16241635, November 2014.

[11] T. Kosaka, S. Elliott, and C. Boucher, "A novel frequency domain filtered-x LMS algorithm for active noise reduction," in Proc. IEEE ICASSP 1997, vol. 1, Munich, Germany, April 1997, pp. 403-406.

[12] H. Buchner, S. Spors, and W. Kellermann, "Wave-domain adaptive filtering: Acoustic echo cancellation for full-duplex systems based on wave-field synthesis," in Proc. IEEE ICASSP 2004, vol. 4, Montreal, Quebec, Canada, May 2004, pp. iv117-iv120.

[13] M. Schneider and W. Kellermann, "A wave-domain model for acoustic mimo systems with reduced complexity," in Proc. IEEE HSCMA, Edinburgh, UK, May 2011, pp. 133 - 138
[14] — - "Adaptive listening room equalization using a scalable filtering structure in the wave domain," in Proc. IEEE ICASSP 2012, Kyoto, Japan, March 2012, pp. 13-16.

[15] S. Spors, H. Buchner, and R. Rabenstein, "A novel approach to active listening room compensation for wave field synthesis using wave-domain adaptive filtering," in Proc. IEEE ICASSP 2004, vol. 4, Montreal, Quebec, Canada, May 2004, pp. iv29iv32.

[16] T. Betlehem and T. D. Abhayapala, "Theory and design of sound field reproduction in reverberant rooms," Journal of the Acoustical Society of America, vol. 117, no. 4, pp. 2100-2111, April 2005.

[17] S. Spors, H. Buchner, R. Raebnstein, and W. Herbordt, "Active listening room compensation for massive multichannel sound reproduction systems," Journal of the Acoustical Society of America, vol. 122, no. 1, pp. 354-369, July 2007.

[18] D. Talagala, W. Zhang, and T. Abhayapala, "Efficient multichannel adaptive room compensation for spatial soundfield reproduction using a modal decomposition," IEEE/ACM Transactions on Audio, Speech, and Language Processing, vol. 22, no. 10, pp. 1522-1532, October 2014.

[19] T. D. Abhayapala and Y. Wu, "Spatial soundfield reproduction with zones of quiet," in Proc. 127th Audio Engineering Society Convention, New York, USA, October 2009.

[20] T. Betlehem, W. Zhang, M. Poletti, and T. Abhayapala, "Personal sound zones: Delivering interface-free audio to multiple listeners," IEEE Signal Processing Magazine, vol. 32, no. 2, pp. 81-91, March 2015.

[21] Y. Wu and T. Abhayapala, "Spatial multizone soundfield reproduction: Theory and design," IEEE Transactions on Audio, Speech, and Language Processing, vol. 19, no. 6, pp. 17111720, August 2011.

[22] S. Spors and H. Buchner, "Efficient massive multichannel active noise control using wave-domain adaptive filtering," in Proc. 3rd International Symposium on Communications, Control and Signal Processing, St. Julians, Malta, March 2008, pp. 1480-1485.

[23] R. Kennedy, P. Sadeghi, T. Abhayapala, and H. M. Jones, "Intrinsic limits of dimensionality and richness in random multipath fields," IEEE Transactions on Signal Processing, vol. 55, no. 6, pp. 2542-2556, June 2007.

[24] T. Abhayapala, T. Pollock, and R. Kennedy, "Characterization of 3D spatial wireless channels," in Vehicular Technology Conference, 2003. VTC 2003-Fall. 2003 IEEE 58th, vol. 1, October 2003, pp. 123-127.

[25] B. Widrow, J. McCool, and M. Ball, "The complex LMS algorithm," Proceedings of the IEEE, vol. 63, no. 4, pp. 719-720, April 1975. 